\title{
Structural Model Correlation Using Large Admissible Perturbations in Cognate Space
}

\author{
Michael M. Bernitsas* and Ricky L. Tawekal† \\ University of Michigan, Ann Arbor, Michigan 48109
}

\begin{abstract}
A nonlinear perturbation method is developed to solve the problem of correlating a finite element model (FEM) to a structure for which an incomplete set of natural frequencies and mode shapes and/or some static deflections have been measured. The solution algorithm can handle differences between FEM and structure, in design variables and response, as large as $100-300 \%$, depending on the scale of the structure and correlation measures. This is achieved incrementally by making inadmissible predictions, identifying the modal cognate space relevant to the correlation measures, and making admissible corrections in the cognate space. The developed computer code postprocesses results of the FEM modal and/or static analyses of the initial model only. No additional finite element analysis is required. Lagrange multipliers reveal the dominant correlation requirements and the active admissible cognate subspace. Depending on the number of correlation variables and measures, an optimal, a unique, or an inadmissible minimal error solution may be produced. Beam and offshore tower examples are used to test the algorithm and investigate conflicting requirements, definition of admissible cognate space, limits of allowable differences between FEM and structure, accuracy, and cost of the nonlinear perturbation method.
\end{abstract}

\section{Nomenclature}

$c_{i j}=$ admixture coefficient for participation of the $j$ th mode to changes in the $i$ th mode

$c_{\mu_{i}}, c_{\omega_{i}}, c_{\phi_{k i}}=$ measured values of static deflection $i$, natural frequency $i, k$ th degree of freedom of mode $i$

$E \quad=$ Young's modulus

$J_{B}, J_{S}, J_{T}=$ set of cognate modes in bending, stretching, torsion

$J_{C} \quad=$ set of modes cognate to modes in $J_{M}$

$J_{C_{i}} \quad=$ set of modes cognate to mode $i$;

$i=1,2, \ldots, n_{r}$

$J_{M} \quad=$ set of modes for which measurements are available

$K_{i} \quad=i$ th component of [ $\left.K \backslash\right]$

$\left[k_{e}\right]=$ stiffness matrix of element, or group of elements, related to property $e$

$[k],\left[{ }^{\prime} K\right]=$ global and generalized stiffness matrices

$\ell=$ index denoting quantity in increment $\ell$; $\ell=1,2, \ldots, N$

$M_{i} \quad=i$ th component of [ $\left.M \backslash\right]$

$\left[m_{e}\right] \quad=$ mass matrix of element, or group elements related to property $e$

$[m],\left[M_{\backslash}\right]=$ global and generalized mass matrices

$N \quad=$ number of increments in predictor-corrector algorithm

$n \quad=$ number of degrees of freedom in FEM

$n_{r} \quad=$ number of extracted structural modes used in RESTRUCT

$p \quad=$ number of correlation variables

$S, S_{a} \quad=$ number of correlation and admissibility constraints

$S_{u}, S_{\omega}, S_{\phi} \quad=$ number of displacement, frequency, and modal node correlation measurements

Received Nov. 7, 1989; revision received Sept. 4, 1990; accepted for publication Sept. 20, 1990. Copyright (C) 1990 by the American Institute of Aeronautics and Astronautics, Inc. All rights reserved.

*Professor, Department of Naval Architecture and Marine Engineering.

†Graduate Student, Research Assistant, Department of Naval Architecture and Marine Engineering.

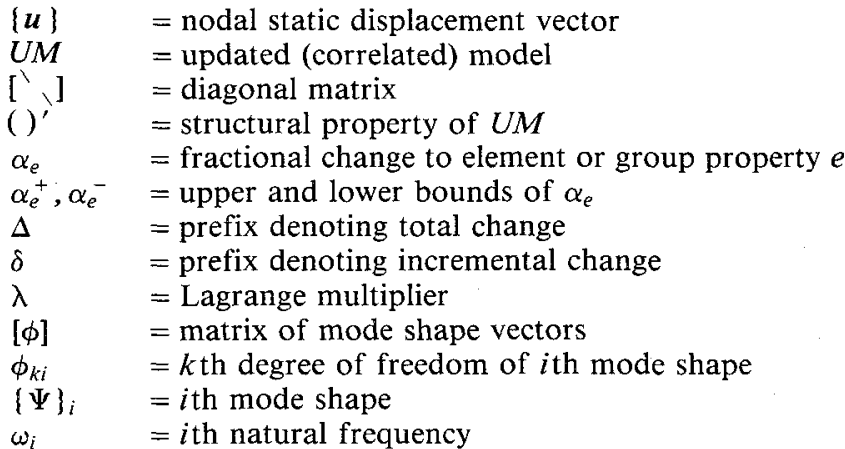

\section{Introduction and Background}

I $\mathrm{N}$ the process of generating a numerical model for a real structure, information is lost in two stages. First, in deriving a mathematical model for the structure, and second, in developing a numerical model from the analytical one. Uncertainty, ignorance, and simplifying assumptions to facilitate modeling are major sources of error. Thus, numerical prediction of static and/or dynamic response of a structure is often inaccurate. The larger manufacturing tolerances are, the greater is the discrepancy between predicted and measured response. This problem is of particular importance and difficulty in finite element analysis (FEA) of marine structures. Offshore structures and ships have large manufacturing tolerances and structural imperfections that make finite element modeling (FEM) and numerical predictions of response highly inaccurate. Differences between predicted and measured response are very big. Model correlation is the process of finding corrected values of the design variables in the FE model of a physical structure, so that numerical predictions of measured response are satisfactory. This can be achieved in a variety of ways, which will be discussed at the end of this paper.

The model correlation problem has been studied extensively for aerospace structures built to tolerances smaller than those of marine structures. In spite of that, aerospace structures also show large discrepancies between measured and predicted response. Mode survey tests ${ }^{1}$ and other measurements are needed to adjust numerical models. $\mathrm{Kabe}^{2}$ used measured modal data to adjust the stiffness matrix and preserved element connectivity. ${ }^{3}$ Baruch $^{4}$ used an optimization procedure 
to currect stiffness and flexiblity matrices. Wei and Zhang ${ }^{5}$ and Berman ${ }^{6}$ corrected the mass matrix based on measured modes, and Taylor ${ }^{7}$ updated the stiffness matrix. Caesar and Peter, ${ }^{8}$ Berman and Nagy, ${ }^{1}$ Chen and Garba, ${ }^{9}$ and Chen and Fuh $^{10}$ updated both stiffness and mass matrices. In all these publications, small differences were allowed between the initial (IM) and updated models (UM) in response and structural properties. The resulting problem was solved by optimization, ${ }^{1-9,11,12}$ with a minimum change criterion if the number of design variables was greater than the number of orthogonality constraints. If the opposite held, the error in satisfaction of constraints was minimized by the generalized inverse ${ }^{10}$ or least squares method. ${ }^{8}$ Collins et al. ${ }^{13}$ used a statistical method for structure identification and minimized a function of the variances and covariances of differences in modal properties and design parameters between measured and predicted responses. In the aforementioned publications, it was assumed that measured mode shapes were exact and the mass and/or stiffness matrices were updated. In an alternative approach, Targoff ${ }^{11}$ and Baruch and Bar Itzhack ${ }^{12}$ assumed that the mass matrix was correct and modified modal vibration measurements to satisfy orthogonality. Modification of the mass matrix ${ }^{1,3,6-10}$ resulted in an updated matrix that did not represent a real structure's mass distribution. Wei and Zhang ${ }^{5}$ remedied this problem by modifying structural elements instead of terms in the mass matrix.

The correlation procedure followed in this paper is diagrammatically shown in Fig. 1. The developed perturbation method and solution algorithm have the following features:

1) Natural frequencies, mode shapes, and static deflections are measured and assumed to be exact.

2) The set of measured modal properties may be incomplete.

3) Differences between IM and UM in response and structural properties up to $100-300 \%$ are allowed, depending on the scale of the structure and the correlation requirements.

4) Both mass and stiffness matrices are updated.

5) Structural element properties, rather than matrix elements, are changed. Thus, the updated matrices represent mass and stiffness matrices of a real structure.

6) IM and UM may differ significantly in the value of elemental stiffness, mass, and geometric properties. Nonetheless, the correlation problem is solved by postprocessing results of only one FEA-that of IM.

7) A nonlinear perturbation algorithm is used that performs linear inadmissible predictions and nonlinear admissible corrections. Perturbations are defined as admissible if they satisfy both stiffness and mass orthogonality, thus ensuring that intermediate (incremental) modes correspond to the real structure calculated as per item 5 .

8) No trial and error or iterations are performed within each increment or anywhere else in the algorithm.

9) At each increment, the correlation problem is solved by minimizing the Euclidian norm of the correlation variables or the error in the constraints, depending on whether the problem is underdetermined or overdetermined, respectively. In general, the former yields better results.

10) To reduce the number of constraints and make the problem underdetermined if possible-for a given number of correlation variables-the number of orthogonality constraints is reduced by identifying space of modes cognate to the correlation modal objectives (measurements).

Linear perturbation methods were first developed by Stetson $^{14,15}$ for structural redesign, allowing for small differences in response, stiffness, and geometric properties between baseline and objective designs. Sandstrom and Anderson ${ }^{16}$ improved that method. Nonlinear perturbation methods were developed by Hoff et al., ${ }^{17}$ Hoff and Bernitsas, ${ }^{18,19}$ Bernitsas et al., ${ }^{20}$ and $\mathrm{Kim}$ and Anderson, ${ }^{21}$ with the main objective being to improve the algorithm and make it applicable to large scale structures and for larger differences between baseline and objective structures. Gans and Anderson ${ }^{22}$ used nonlinear perturbation methods in optimization of turbine blades by

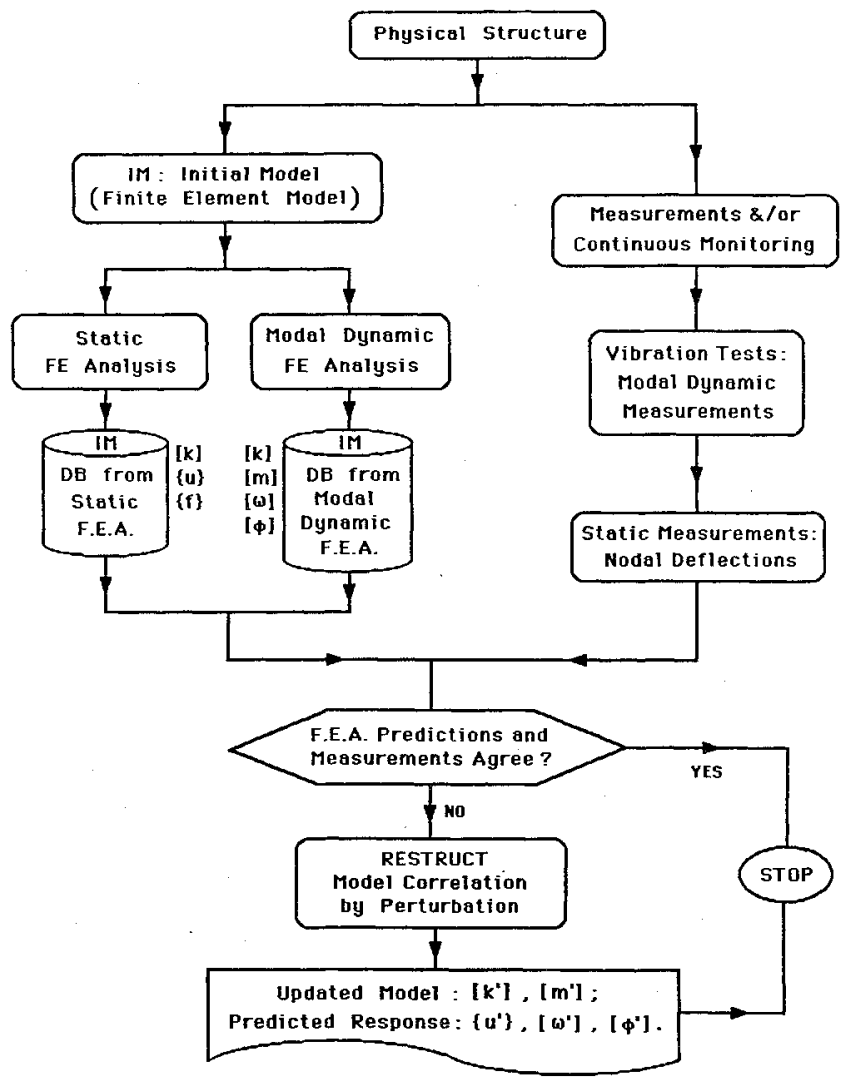

Fig. 1 FEA predictions, response measurements, model correlation.

incorporating centrifugal and corriolis effects. They perform, however, a FEA by MSC/NASTRAN at each increment. Kim and Bernitsas ${ }^{23}$ introduced static deflections as redesign objectives along with modal measurements. Other approaches to redesign have been developed by Hafka and Prasad ${ }^{24}$ and Haug et al. ${ }^{25}$

The problem of model correlation by perturbation is defined in Sec. II. In Sec. III, a nonlinear incremental perturbation algorithm for model correlation is introduced. Implementation of the algorithm in computer code RESTRUCT (REdesign of STRUCTures), ${ }^{26}$ which serves as postprocessor to special and general purpose FE codes, including MSC/ NASTRAN, is discussed also in this section. In the remaining three sections, a five-element clamped-hinged beam and a 104-element 192-DOF offshore tower are used to study correlation in bending, in torsion and in simultaneous bending and torsion. Cognate space identification, conflict in correlation requirements, activity of admissiblity constraints, and limitations on differences between IM and UM in response and structural properties are emphasized.

\section{Model Correlation by Perturbation}

Figure 1 shows that model correlation is performed in this paper with respect to static and/or modal dynamic measured response properties. The general perturbation static and free vibration equations which relate UM to IM are first derived in Sec. II.A. Then the model correlation problem is defined in Sec. II.B.

\section{A. General Perturbation Equations}

The initial and updated models (IM and UM) are related through the following perturbation equations ${ }^{14,16,18}$ for stiffness matrix $[k]$, static deflection vector $\{\boldsymbol{u}\}$, mass matrix $[m]$, natural frequencies $\omega$, and modal matrix $[\phi]$, respectively:

$$
\left[k^{\prime}\right]=[k]+[\Delta k]
$$




$$
\begin{gathered}
\left\{\boldsymbol{u}^{\prime}\right\}=\{\boldsymbol{u}\}+\{\Delta u\} \\
{\left[m^{\prime}\right]=[m]+[\Delta m]} \\
{\left[{ }^{\prime} \omega^{\prime 2} \backslash\right]=\left[\omega^{2} \backslash\right]+\left[{ }^{\prime} \Delta\left(\omega^{2}\right) \backslash\right]} \\
{\left[\phi^{\prime}\right]=[\phi]+[\Delta \phi]}
\end{gathered}
$$

where primed and unprimed quantities refer to UM and IM, respectively, $\left[{ }^{\prime} \omega^{2}\right]$ is the diagonal matrix of the eigenvalues, prefix $\Delta$ indicates differences between IM and UM, $[\phi]=$ $\left[\{\Psi\}_{1},\{\Psi\}_{2}, \ldots,\{\Psi\}_{n}\right]$, and $\{\Psi\}_{j}$ is the $j$ th eigenvector corresponding to the $j$ th eigenvalue $\omega_{j}^{2}$, defined by the free vibration equilibrium equation

$$
\left([k]-\omega_{j}^{2}[m]\right)\{\Psi\}_{j}=\{0\}, \quad \text { for } j=1,2, \ldots, n
$$

where the $n$ eigenvalues $\omega_{j}, j=1,2, \ldots, n$, satisfy equation

$$
\operatorname{det}\left([k]-\omega^{2}[m]\right)=0
$$

In Eq. (6), only Rayleigh's damping can be introduced. The corresponding energy balance equation for IM is

$$
\left.\left[{ }^{\prime} K_{\backslash}\right]=\left[{ }^{\prime} M_{\backslash}\right]\left[{ }^{\prime} \omega^{2}\right]\right]
$$

where [ $K_{\backslash}$ ] and [ $\left.M_{\backslash}\right]$ are the generalized diagonal stiffness and mass matrices. Relations similar to Eqs. (6-8) hold for UM, with

$$
\begin{aligned}
& {\left[{ }^{\prime} K^{\prime} \backslash\right]=\left[{ }^{\prime} K_{\backslash}\right]+\left[{ }^{\prime} \Delta K_{\backslash}\right]} \\
& {\left[{ }^{\prime} M^{\prime}\right]=\left[{ }^{\prime} M_{\backslash}\right]+\left[{ }^{\prime} \Delta M_{\backslash}\right]}
\end{aligned}
$$

Introducing these relations and Eqs. (1-5) into Eq. (8), we derive the general perturbation equation in matrix form:

$$
\begin{aligned}
& {\left[\phi^{\prime}\right]^{T}[\Delta k]\left[\phi^{\prime}\right]-\left[\phi^{\prime}\right]^{T}[\Delta m]\left[\phi^{\prime}\right]\left[\omega^{\prime}{ }_{\backslash}\right]} \\
& =\left[\phi^{\prime}\right]^{T}[m]\left[\phi^{\prime}\right]\left[\omega^{\prime}{ }^{2}\right]-\left[\phi^{\prime}\right]^{T}[k]\left[\phi^{\prime}\right]
\end{aligned}
$$

In scalar form, Eq. (9) is equivalent to

$$
\begin{gathered}
\left.\left\{\Psi^{\prime}\right\}_{j}^{T}[\Delta k]\left\{\Psi^{\prime}\right\}_{i}-\omega^{\prime 2} i \Psi^{\prime}\right\}_{j}^{T}[\Delta m]\left\{\Psi^{\prime}\right\}_{i} \\
=\left\{\Psi^{\prime}\right\}_{j}^{T}[m]\left\{\Psi^{\prime}\right\}_{i} \omega_{i}^{\prime 2}-\left\{\Psi^{\prime}\right\}_{j}^{T}[k]\left\{\Psi^{\prime}\right\}_{i}, \\
\qquad \text { for } i=j=1,2, \ldots, n \\
\left\{\Psi^{\prime}\right\}_{j}^{T}[\Delta k]\left\{\Psi^{\prime}\right\}_{i}=-\left\{\Psi^{\prime}\right\}_{j}^{T}[k]\left\{\Psi^{\prime}\right\}_{i}, \quad \text { for } i \neq j \\
\left\{\Psi^{\prime}\right\}_{j}^{T}[\Delta m]\left\{\Psi^{\prime}\right\}_{i}=-\left\{\Psi^{\prime}\right\}_{j}^{T}[m]\left\{\Psi^{\prime}\right\}_{i}, \quad \text { for } i \neq j
\end{gathered}
$$

where, in Eqs. (11) and (12), $i=1,2, \ldots, n$ and $j=i+1$, $i+2, \ldots, n$. The corresponding static general perturbation equation is

$$
\{\Delta \boldsymbol{u}\}=-([k]+[\Delta k])^{-1}[\Delta k]\{\boldsymbol{u}\}
$$

where it is assumed that static nodal force vector $\{f\}$ is the same for IM and UM, that is $\{f\}=\left\{f^{\prime}\right\}$.

\section{B. Problem Definition}

Note that $[\Delta k]$ and $[\Delta m]$ represent structural perturbations that must be computed based on some measured response properties $\left\{u^{\prime}\right\},\left[{ }^{\prime} \omega^{2}\right]$, and $\left[\phi^{\prime}\right]$. In doing so, element connectivity must be preserved. Further, correlation values, which are defined below, are bounded by practical limits defined by inequality (23).

\section{Correlation Variables}

Let $\alpha_{e}, e=1,2, \ldots, p$ be the fractional change in property $e$, where $p$ properties of elements, or groups of elements, are allowed to change. Such properties may be mass, bending stiffness, torsional stiffness, etc., ${ }^{17}$ or geometric properties like linear dimensions of a beam cross section, ${ }^{18}$ plate thickness, ${ }^{23}$ and tube internal and external diameters. ${ }^{20}$ Several elements may be linked together in one group to ensure that they remain identical in UM..$^{27}$ More than one property may be allowed to change in each element or group of elements. Then, changes in global stiffness and mass matrices can be expressed as summation of all changes as

$$
\begin{aligned}
& {[\Delta k]=\sum_{e=1}^{p}\left[\Delta k_{e}\right]=\sum_{e=1}^{p}\left[k_{e}\right] \alpha_{e}} \\
& {[\Delta m]=\sum_{e=1}^{p}\left[\Delta m_{e}\right]=\sum_{e=1}^{p}\left[m_{e}\right] \alpha_{e}}
\end{aligned}
$$

where $\left[k_{e}\right]$ and $\left[m_{e}\right]$ are the stiffness and mass matrices of the $e$ th element or group of elements. It should be noted that several $\alpha_{e}$ may refer to the same element but different properties, like bending, torsion, stretching, etc. Any $\alpha_{e}$ may correspond to a property that may affect only $\left[k_{e}\right]$ or $\left[m_{e}\right]$.

\section{Correlation Measures}

All measured modal and static deflection properties can be used as correlation measures (requirements). Those are defined by the following equations:

$$
\begin{array}{rr}
u_{j}^{\prime}=u_{j}+\Delta u_{i}=c_{u_{i}}, & i=1,2, \ldots, S_{u} \\
\omega_{i}^{\prime 2}=\omega_{i}^{2}+\Delta \omega_{i}^{2}=c_{\omega_{i}}, & i=1,2, \ldots, S_{\omega} \\
\phi_{k i}^{\prime}=\phi_{k i}+\Delta \phi_{k i}=c_{\phi_{k i}}, & \text { number of }(k, i)=S_{\phi}
\end{array}
$$

where the right-hand sides are the measured quantities that are assumed exact and are used as correlation measures, and $S_{\mu}$, $S_{\omega}, S_{\phi}$ are the numbers of static deflection, frequency, and modal measures, respectively.

\section{Problem Formulation}

The general perturbation equations (10-13) can be expressed as Eqs. (19-22), respectively, in terms of correlation variables $\alpha_{e}, e=1,2, \ldots, p$, using Eqs. (14) and (15).

$$
\begin{aligned}
& \sum_{e=1}^{p}\left(\left\{\Psi^{\prime}\right\}_{i}^{T}\left[k_{e}\right]\left\{\Psi^{\prime}\right\}_{i}-\omega_{i}^{\prime 2}\left\{\Psi^{\prime}\right\}_{i}^{T}\left[m_{e}\right]\left\{\Psi^{\prime}\right\}_{i}\right) \alpha_{e} \\
& =\omega_{i}^{\prime 2}\left\{\Psi^{\prime}\right\}_{i}^{T}[m]\left\{\Psi^{\prime}\right\}-\left\{\Psi^{\prime}\right\}_{i}^{T}[k]\left\{\Psi^{\prime}\right\}_{i}, \\
& i=1,2, \ldots, n \\
& \sum_{e=1}^{p}\left\{\Psi^{\prime}\right\}_{j}^{T}\left[k_{e}\right]\left\{\Psi^{\prime}\right\}_{i} \alpha_{e}=-\left\{\Psi^{\prime}\right\}_{j}^{T}[k]\left\{\Psi^{\prime}\right\}_{i}, \\
& \quad \text { for } i=1,2, \ldots, n, \quad j=i+1, i+2, \ldots, n \\
& \sum_{e=1}^{p}\left\{\Psi^{\prime}\right\}_{j}^{T}\left[m_{e}\right]\left\{\Psi^{\prime}\right\}_{i} \alpha_{e}=-\left\{\Psi^{\prime}\right\}_{j}^{T}[m]\left\{\Psi^{\prime}\right\}_{i}, \\
& \quad \text { for } i=1,2, \ldots, n, \quad j=i+1, i+2, \ldots, n \\
& \quad\{\Delta u\}=-\sum_{e=1}^{p}\left([k]+\sum_{e=1}^{p}\left[k_{e}\right] \alpha_{e}\right)^{-1}\left[k_{e}\right]\{u\} \alpha_{e}
\end{aligned}
$$

The perturbation correlation problem can now be defined as follows: Define UM by computing the values of correlation variables $\alpha_{e}, e=1,2, \ldots, p$, to satisfy $S_{u}$ static deflection, $S_{\omega}$ natural frequency, and $S_{\phi}$ modal measures defined by Eqs. (16-18), respectively. Practical constraints in the form of lower and upper bounds may be imposed on the correlation variables:

$$
-1<\alpha_{e}^{-} \leq \alpha_{e} \leq \alpha_{e}^{+}, \quad e=1,2, \ldots, p
$$


Further, the number of orthogonality conditions (20) and (21) imposed on $\alpha_{e}$ is no greater than

$$
S_{a}=S_{\omega}\left[(2 n-1)-S_{\omega}\right]
$$

where it is assumed that eigenvectors are measured only when the corresponding natural frequency is also measured. Those are the admissibility conditions that, when imposed, ascertain that $\left[k^{\prime}\right],\left[m^{\prime}\right],\left\{u^{\prime}\right\},\left[\phi^{\prime}\right]$, and $\left[\omega^{\prime}\right]$ correspond to the same real structure. The remaining orthogonality conditions need not be imposed on $\alpha_{e}$, since they do not introduce any additional correlation requirements. Instead, they can be used to compute the eigenvectors of UM, which have not been measured. In the algorithm developed in the next section, the admissible domain is further reduced to a cognate mode admissible space. In the latter, an active subspace is identified with the aid of the Lagrange multipliers computed in the numerical solution.

Solution of the above mathematical model presents the following difficulties:

1) Differences in response between IM and UM are large; thus, $\alpha_{e}$ is expected to be large. If $\alpha_{e}$ corresponds to linear dimensions of elements, such as plate thickness, shaft diameter, or height of rectangular beam cross section, nonlinear expressions may result for $[\Delta k]$ and $[\Delta m]$ instead of Eqs. (14) and (15).

2) For an incomplete set of measurements, unknown modal properties are involved in Eqs. (19-21), and unknown deflections are involved in Eq. (22). Those are functions of $\alpha_{e}$, and consequently, the generalized perturbation Eqs. (19-22) become implicit nonlinear expressions of correlation variables.

3) The matrix inversion that appears in Eq. (22) must be avoided because it is expensive and inaccurate for large-scale structures.

4) Depending on the relation between the number of correlation variables $p$, the number of equality constraints $S=S_{u}+S_{\omega}+S_{\phi}+S_{\alpha}$, and the $2 p$ bounds on the correlation variables defined by Eq. (23), the feasible domain may or may not be empty. In the former case, a minimum error solution can be produced; in the latter, an optimality criterion is needed.

5) For structures with small manufacturing tolerances, there may be confidence in the total mass estimate. In such a case, an additional constraint can be imposed on the correlation variables. ${ }^{5}$

\section{Numerical Solution}

The model correlation perturbation problem formulated in Sec. II-Eqs. (16-23) -is solved incrementally using a prediction/correction scheme. The incremental algorithm is developed in Sec. III.A. The linear and nonlinear solvers ued in RESTRUCT to solve the resulting prediction and correction problems at each increment are described in Sec. III.B. Computer implementation of the algorithm in RESTRUCT is explained in Sec. III.C.

\section{A. Incremental Formulation}

The correlation measures $c_{u_{i}}, i=1,2, \ldots, S_{u} ; c_{\omega_{i}}, i=$ $1,2, \ldots, S_{\omega}$; and $S_{\phi}, c_{\phi_{k i}}$, specified in Eqs. (16-18), will be achieved in increments no larger than $7 \%$. The purpose of this is to keep correlation variables $\alpha_{e}, e=1,2, \ldots, p$ small in each increment. Of course, this requires introduction of bounds on incremental $\alpha_{e}$. Then, Eqs. (19-22), which are implicit expressions of $\alpha_{e}$, can be linearized to produce a satisfactory linear prediction. Let $\ell=1,2, \ldots, N$ indicate the th increment and $N$ the total number of increments. Let prefix $\delta$ denote difference in any increment, as $\Delta$ does in the complete problem. Then, the incremental model correlation problem is defined by the counterparts of Eqs. (16-22) and

$$
-1<_{p} \alpha_{e}^{-} \leq{ }_{p} \alpha_{e} \leq{ }_{\ell} \alpha_{e}^{+}, \quad e=1,2, \ldots, p
$$

where

$$
\alpha_{e}^{-}=-0.15, \quad{ }_{p} \alpha_{e}^{+}=+0.15, \quad\left(1+\alpha_{e}\right)=\prod_{\ell=1}^{N}\left(1+{ }_{p} \alpha_{e}\right)
$$

The $7 \%$ upper bound on incremental correlation measures and the $15 \%$ bound imposed on the incremental correlation variables by condition (25) permit linearization of perturbation equations (19-22). This is done in the prediction phase of the algorithm. The process of model correlation as performed by RESTRUCT is diagramatically shown in Fig. 2. The three phases of the incremental algorithm described below-that is prediction, cognate space identification, and correction-are summarized in Fig. 3.

\section{Inadmissible Linear Prediction}

Following the linear perturbation algorithm developed by Stetson ${ }^{14}$ and Stetson and Harrison ${ }^{15}$ and improved by Sandström and Anderson, ${ }^{16}$ we have

$$
\left[\delta_{\ell} \phi\right]={ }_{p}[\phi]_{\ell}[c]^{T}
$$

where ${ }_{p}[c]$ is matrix of admixture coefficients, ${ }_{\ell} c_{i i}=0,{ }_{\ell} c_{i j}$, $i, j=1,2, \ldots, n_{r}$ are small, and $n_{r}$ is the number of extracted modes used in the algorithm:

$$
\begin{gathered}
\delta_{\ell} \omega_{i}^{2}=\frac{1}{{ }_{p} M_{i}}\left[\sum _ { e = 1 } ^ { p } \left({ }_{\ell}(\Psi\}_{i}^{T}\left[k_{e}\right]_{\ell}\{\Psi\}_{i}\right.\right. \\
\left.\left.-{ }_{p} \omega_{i \ell}^{2}\{\Psi\}_{i}^{T}\left[m_{e}\right]_{p}\{\Psi\}_{i}\right)_{\ell} \alpha_{e}\right]=\delta_{\ell} c_{\omega_{i}} \\
i=1,2, \ldots, S_{\omega} \\
{ }_{\ell} c_{i j}=\sum_{e=1}^{p} \frac{1}{{ }_{\ell} M_{j}\left(p \omega_{i}^{2}-{ }_{p} \omega_{j}^{2}\right)}\left({ }_{\ell}\{\Psi\}_{j}^{T}\left[k_{e}\right]_{\ell}\{\Psi\}_{i}\right. \\
-{ }_{p} \omega_{i}^{2}\left\{\{\Psi\}_{j}^{T}\left[m_{e}\right]_{\ell}\{\Psi\}_{i}\right)_{\ell} \alpha_{e}
\end{gathered}
$$

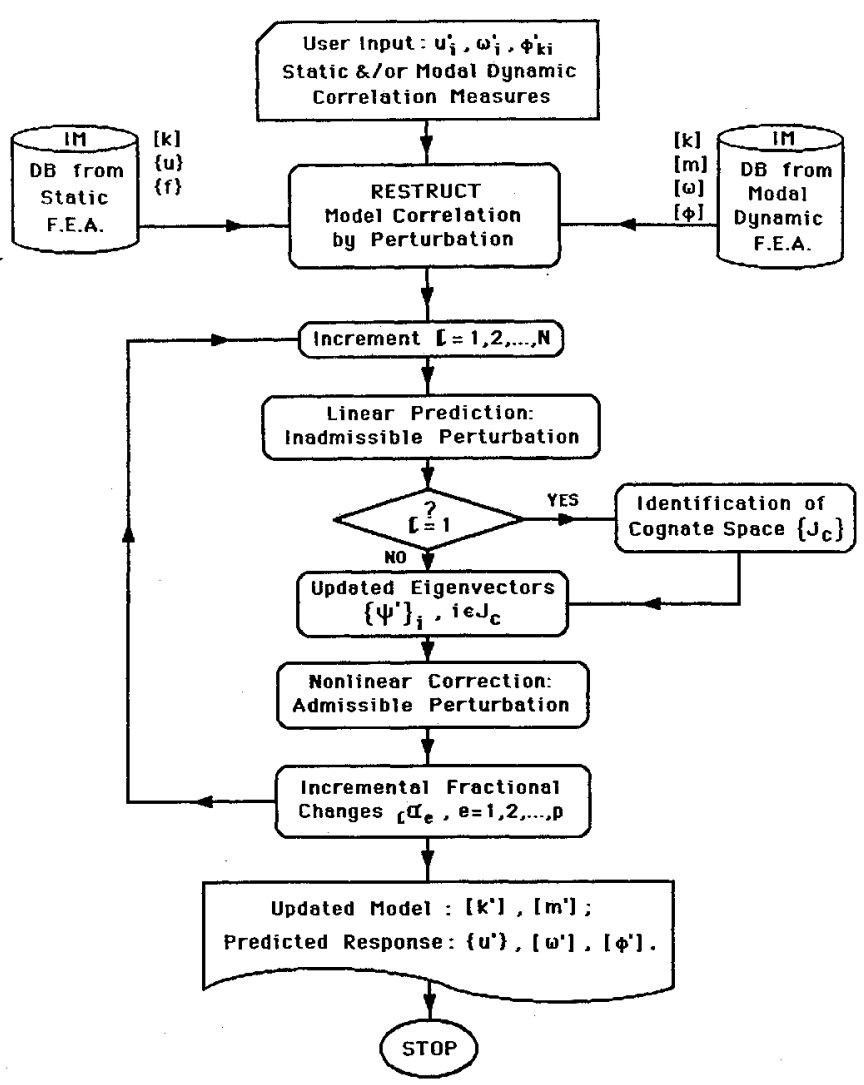

Fig. 2 Finite element model correlation by RESTRUCT. 
INCREMENT

\begin{tabular}{|c|c|c|}
\hline & $\begin{array}{l}\text { PHASE \# : Inadmissible } \\
\text { Lineor Prediction }\end{array}$ & $\begin{array}{c}\text { PAASE \#Z : Admissible } \\
\text { Nonlinear Correction }\end{array}$ \\
\hline $\begin{array}{l}\text { Perturbation } \\
\text { Equations }\end{array}$ & $\delta \omega_{i}^{2},(27): \delta \Phi_{k j} \cdot(29)$ & $\omega_{i}^{2},(33) ; u_{i}^{\prime},(36)$ \\
\hline $\begin{array}{l}\text { Correlation } \\
\text { Requirements }\end{array}$ & 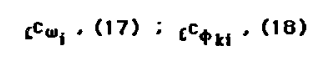 & ${ }^{c} c_{u_{i}},(16):{ }_{\left[w_{i}\right.},(17)$ \\
\hline Input : & 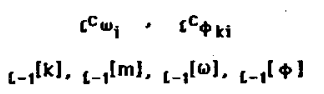 & 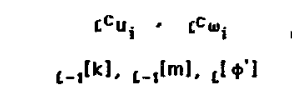 \\
\hline Unknowns & $\boldsymbol{c}_{\boldsymbol{c}}^{\boldsymbol{\alpha}_{e}}$, & ,...,p \\
\hline $\begin{array}{l}\text { Number of } \\
\text { Canstraints }\end{array}$ & $s_{\omega}+s_{\Phi}:(17),(27)$ & $\begin{array}{l}S_{u}+S_{w}+S_{0}:(17),(27) \\
(34),(35) \\
(25)\end{array}$ \\
\hline $\begin{array}{l}\text { Solution } \\
\text { Method }\end{array}$ & $\begin{array}{l}\text { Infinite Solutions: Optimiz } \\
\text { criteri } \\
\text { Unique Solution: Linear eq } \\
\text { No Solution: Minimum err } \\
\text { constraints; }\end{array}$ & $\begin{array}{l}\text { ion with minimum change } \\
\mathrm{C}_{e} \cdot \mathrm{s} \text { within bounds } \\
\text { ion solver } \\
\text { in satisfaction of equality } \\
\text { neralized Inverse }\end{array}$ \\
\hline Output & $\begin{array}{l}\text { Linear predictions } \\
\qquad \begin{array}{l}\mathbb{C}_{\mathrm{e}}, \mathrm{e} \\
\mathfrak{l}^{[c]},(28) \\
\mathfrak{d}^{[\delta \phi]},(26) \\
{ }_{\mathfrak{l}}^{\left[\phi^{\prime}\right]},(5)\end{array}\end{array}$ & 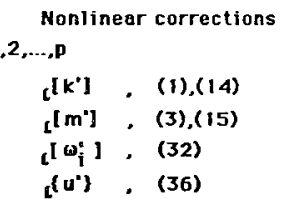 \\
\hline $\begin{array}{l}\text { Cognate } \\
\text { Space }\end{array}$ & $\begin{array}{l}\text { PHASE \#2 = for } C=1 \text {, } \\
\text { Cognate Space identification }\end{array}$ & $\begin{array}{l}\text { Active Admissible } \\
\text { Cognate Subspace }\end{array}$ \\
\hline
\end{tabular}

Fig. 3 Algorithm for structural model correlation, using large admissible perturbations in cognate subspaces.

where Eqs. (27) and (28) represent the linearized diagonal and offdiagonal terms, respectively, in the incremental difference in energy balance between ${ }_{q} I M$ and ${ }_{2} U M$;

$$
\delta_{\ell} \phi_{k i}=\sum_{j=1, j \neq i}^{n_{r}} \phi_{k j \ell} c_{i j}=\delta_{\ell} c_{\phi_{k i}}, \quad \text { number of }(k, i)=S_{\phi}
$$

which is derived directly from Eq. (26), and where an entire mode or only some modal degrees of freedom (DOFs) have been measured.

The prediction problem can be stated as follows: Calculate the incremental correlation variables $\alpha_{e}, e=1,2, \ldots, p$ subject to the incremental counterparts of Eqs. (27), (29), and (25). This problem may be underdetermined or overdetermined. (The solvers used in both cases are described in Sec. III.B.) At the end of the prediction phase, Eq. (29) is used to compute mode shapes that were not measured in vibration tests. Partially measured modes are also completed by computations, using Eq. (29). If modes are not completed, orthogonality would be grossly violated. It should be emphasized that linear predictions are inadmissible because orthogonality conditions (20) and (21) are not satisfied exactly. The $n_{r}\left(n_{r}-1\right)$ equations (28) that are satisfied instead are necessary and sufficient conditions. It is their linearized versions, however, and not Eqs. (20) and (21), that are satisfied implicitly by using Eq. (28) to compute the incremental admixture coefficients. In the numerical applications (see Secs. IV, V, and VI), the Lagrange multipliers in the correction phase corresponding to orthogonality conditions (20) and (21) are small compared to those for Eqs. (19) and (22). This implies that violation of admissibility in prediction is minor. Even though the predicted ${ }_{\ell}\left[k^{\prime}\right]$ and ${ }_{\ell}\left[m^{\prime}\right]$ correspond to a real structure-because we allow structural elements and not individual matrix terms to change-the step made in the prediction phase toward solution is inadmissible, because orthogonality is violated. In the correction phase, this situation will be remedied.

\section{Cognate Space Identification}

In the prediction phase, the admixture coefficients $c_{i j}, i, j=$ $1,2, \ldots, n_{r}$ are computed using Eq. (28). Then, the normal modes that have not been measured are computed using Eqs. (26) or (29). Computation of the admixture coefficients is expensive and may account for as much as $80 \%$ of incremental CPU time. Certain modes, however, may have little or no effect on other modes, in which case the corresponding $c_{i j}$ are very small. The CPU time for computation of $c_{i j}$ and the total CPU time can be reduced drastically by factors as high as 4.5 and 4 , respectively (see Secs. IV and V), if subspaces of cognate modes are identified. For example, in the offshore tower problem studied in Secs. IV, V, and VI, bending eigenvectors define the bending cognate space, and torsional eigenvectors define the torsional cognate space. Admixture coefficients relating modes within the same cognate space have high values. Admixture coefficients relating a mode in a cognate space to a mode outside that space are very small. The latter may be in another cognate space or unclassified.

Cognate modes are identified numerically in the prediction phase of the first increment by computing $c_{m j}$, for $j=$ $1,2, \ldots, n_{r}$, where index $m$ represents only modes for which measured data are available. Admixture coefficients relating cognate modes are several orders of magnitude-between 4 and 8 -greater than those relating noncognate modes. The latter are not computed once they are identified.

The following definitions must be introduced at this point. Let $J_{M}$ be the set of modes for which measurements are available, and $J_{C_{i}}, i=1,2, \ldots, n_{r}$ the set of modes cognate to mode $i$. Obviously, most of those are identical. In fact, in most applications only a few sets of cognate modes are identified, like $J_{B}$ for bending, $J_{T}$ for torsion, $J_{S}$ for stretching. Note that $J_{C}$ is the set of modes cognate to those in $J_{M}$; e.g., $J_{C}$ could be equal to $J_{B}, J_{T}, J_{S}$ or any union of those.

Based on the above definitions, we can state more clearly that

$$
c_{i j}=0, \text { for either } i \notin J_{C} \text { or } j \notin J_{C}
$$

The modal DOFs that have not been measured are computed by modifying the first equation in expression (29) as

$$
\delta_{\ell} \phi_{k i}=\sum_{j \in J_{C_{i}}, j \neq 1} \phi_{k j} \phi_{i j}, \quad \text { for } k=1,2, \ldots, n, \quad i \in J_{C}
$$

\section{Correction into the Admissible Cognate Space}

At this point, from the first two phases in the $\mathrm{lth}$ increment, we have used some of the available information and equations, we have made some predictions and calculations, but we have not reached our incremental goals because we have not used all available measurements and we have not satisfied Eqs. (19-22) in their full implicit nonlinear form. Specifically,

1) Frequency measurements have been used by combining Eqs. (17) and (27). The latter is a linearized expression of energy balance equation (19).

2) Mode shape measurements, whether they represent a complete or incomplete set of modes, and complete or incomplete mode shapes, have been used by combining Eqs. (18) and (29). The latter is a linearized version of the offdiagonal terms in the energy balance expression (9).

3) The static deflection measurements, expressed by Eq. (16), and the corresponding static equilibrium equation (22) have not been used.

4) Set $J_{C}$ has been identified. The mode shapes that have not been measured and the unmeasured DOFs of partially measured modes have been computed combining Eqs. (26), (28), (30), and (31).

5) Of the generalized perturbation equations (19-22), Eq. (22) has not been used, whereas linearized versions of Eqs. (19-21) have been satisfied for the $n_{r}$ extracted modes. 
6) The $\left(n_{r}-S_{\omega}\right)$ natural frequencies that have not been measured are computed using the Rayleigh quotient.

$$
\lambda_{i}^{\prime}=\omega_{i}^{\prime 2}=\frac{K_{i}^{\prime}}{M_{i}^{\prime}}=\frac{\left\{\Psi^{\prime}\right\}_{i}^{T}\left[k^{\prime}\right]\left\{\Psi^{\prime}\right\}_{i}}{\left\{\Psi^{\prime}\right\}_{i}^{T}\left[m^{\prime}\right]\left\{\Psi^{\prime}\right\}_{i}}
$$

A goal in the correction phase is to correct predictions by bringing them back into the admissible cognate space. The problem is defined below. Calculate corrected values of incremental correlation variables $\alpha_{e}, e=1,2, \ldots, p$ so that the following conditions are satisfied:

$$
\begin{aligned}
& \sum_{e=1}^{p}\left({ }_{p}\left\{\Psi^{\prime}\right\}_{i}^{T}\left[k_{e}\right]_{p}\left\{\Psi^{\prime}\right\}_{i}-\omega^{\prime 2}{ }_{i}\left\{\Psi^{\prime}\right\}_{i}^{T}\left[m_{e}\right]_{p}\left\{\Psi^{\prime}\right\}_{i}\right){ }_{p} \alpha_{e} \\
& ={ }_{p} \omega_{i}^{\prime 2}\left\{\left(\Psi^{\prime}\right\}_{i}^{T}[m]_{\ell}\left\{\Psi^{\prime}\right\}_{i}-{ }_{\ell}\left\{\Psi^{\prime}\right\}_{i}^{T}[k]_{\mathcal{R}}\left\{\Psi^{\prime}\right\}_{i}\right. \\
& i \in J_{M}
\end{aligned}
$$

where measured values are defined by Eqs. (17) and (18),

$$
\begin{aligned}
& \sum_{e=1}^{p}\left\{\Psi^{\prime}\right\}_{j}^{T}\left[k_{e}\right]_{\ell}\left\{\Psi^{\prime}\right\}_{i p} \alpha_{e}=-{ }_{\ell}\left\{\Psi^{\prime}\right\}_{j}^{T}[k]_{\ell}\left\{\Psi^{\prime}\right\}_{i} \\
& \text { for } i \in J_{M}, \quad j \in J_{C_{i}} \\
& \sum_{e=1}^{p}\left\{\Psi^{\prime}\right\}_{j}^{T}\left[m_{e}\right]_{\ell}\left\{\Psi^{\prime}\right\}_{i} \alpha_{e}=-{ }_{r}\left\{\Psi^{\prime}\right\}_{j}^{T}[m]{ }_{e}\left\{\Psi^{\prime}\right\}_{i} \\
& \text { for } i \in J_{M}, \quad j \in J_{C_{i}} \\
& \left\{u^{\prime}\right\}_{i}=\sum_{r=1}^{n_{r}} \frac{{ }_{\ell} \phi_{i r}^{\prime} A_{r}}{{ }_{p} B_{r}}-\sum_{e=1}^{p}\left(\sum_{r=1}^{n_{r}} \frac{{ }_{\ell} \phi_{i r}^{\prime} A_{r}}{{ }_{\ell} B_{r}^{2}}{ }_{\ell} C_{r e}\right){ }_{\ell} \alpha_{e}
\end{aligned}
$$

where

$$
\begin{gathered}
{ }_{\ell} A_{r}=\sum_{j=1}^{n}{ }_{\ell} \phi_{j r}^{\prime} f_{j}, \quad{ }_{\ell} B_{r}={ }_{\ell}\left\{\Psi^{\prime}\right\}_{r}^{T}[k]_{\ell}\left\{\Psi^{\prime}\right\}_{r} \\
{ }_{\ell} C_{r e}={ }_{\ell}\left\{\Psi^{\prime}\right\}_{r}^{T}\left[k_{e}\right]{ }_{\ell}\left\{\Psi^{\prime}\right\}_{r}
\end{gathered}
$$

Equation (36) represents the linearized version of Eq. (22) derived by Kim and Bernitsas, ${ }^{23}$ and static deflection measurements are defined by Eq. (16). Finally, upper and lower bound constraints are placed in $\alpha_{e}$, as per Eq. (25).

As in the prediction phase, the correction phase problem may be underdetermined or overdetermined. Solvers used in both cases are described below.

\section{B. Linear and Nonlinear Solvers}

The problems formulated in both the prediction and correction phases in each increment may be overdetermined or underdetermined. In the former case, a generalized inverse algorithm is used ${ }^{10,23}$ to produce a minimum error solution in satisfaction of all equality constraints. The user of computer code RESTRUCT is informed accordingly because it is most likely that condition (25) is violated, which would render predictions unacceptable. The designer, then, must reduce the incremental correlation requirements to satisfy condition (25), or allow more flexibility to the system by introducing additional $\alpha_{e}$. The generalized inverse algorithm uses a simultaneous linear equation solver.

In general, underdetermined problems provide more accurate results. For such problems, an optimality criterion is needed to select the best feasible solution. A minimum change criterion between ${ }_{\ell} I M$ and ${ }_{\ell}$ UM requires minimization of the square of the Euclidean norm of $\alpha_{e}$. Since in both prediction and correction problems, all constraints are linear with respect to $\alpha_{e}$, those problems can be solved by quadratic programming ${ }^{28}$ using QPSOL. ${ }^{29}$ If a linear optimality criterion is used instead, the Simplex algorithm ${ }^{28}$ can be applied. If Eqs. (14) and (15) are replaced by nonlinear expressions, as may be needed for plate ${ }^{18}$ or tubular ${ }^{20}$ elements, a nonlinear programming solver like NPSOL must be used. ${ }^{29}$
In overdetermined problems, all of the admissibility conditions (34) and (35) are violated. In underdetermined problems, all of the admissibility conditions are satisfied. The corresponding Lagrange multipliers produced by QPSOL provide relevant sensitivity information. In general, they show that constraints (33) and (36) dominate the solution. They also show that within a $J_{C}$ set, certain admissibility conditions may be inactive. This is the case, for example, in bending of the offshore tower in Sec. IV.B. In the $J_{B}$ set, orthogonality conditions (34) and (35), relating bending modes in two mutually perpendicular directions, are inactive. This information can be used a priori to reduced computational time.

\section{Computer Implementation}

The algorithm described above is summarized in Fig. 3, and is implemented in code RESTRUCT. ${ }^{26}$ The model correlation process in RESTRUCT is outlined in Fig. 2. That code is about 20,500 Fortran 77 commands and was initially developed to solve the problem of structural redesign. ${ }^{23}$ Currently, it also solves the problem of model correlation described in this paper and the problem of redundancy and failure mode identification. RESTRUCT may accept any kind of stiffness and mass matrices produced by a special or general purpose FE code, including MSC/NASTRAN. At the present stage, however, it can perform computations with concentrated mass, consistently distributed mass, spring, rod, bar, triangular plate, quadrilateral plate, and marine riser tubular elements. Several numerical applications are used in the rest of this paper to test the algorithm, study conflicting requirements, limits of measured and structural differences between IM and UM, and identification of active admissible cognate space. Analysis of IM is performed by MSC/NASTRAN, and results are postprocessed by RESTRUCT. Reanalysis of UM is also done by MSC/NASTRAN in order to assess the accuracy of computations.

\section{Correlation in Bending}

The problem of model correlation in bending is studied in this section, using beam and offshore tower numerical applications. Difference of as much as $100 \%$ between predicted and measured response is assumed. Resulting differences between IM and UM in correlation variables may be as high as $400 \%$. The relative error between response of UM as computed by RESTRUCT, and actual UM response as computed by MSC/ NASTRAN, is calculated in all applications in this and the following two sections. Dependence of relative error and $\mathrm{CPU}$ time on magnitude of correlation measures, conflict in correlation measures, number of extracted modes and admissibility constraints, definition of cognate space and active cognate subspace, are studied.

\section{A. Beam Model}

MSC/NASTRAN is first used to perform modal dynamic and static analysis of the five-element, nine-DOF clampedhinged beam shown in Fig. 4. For the beam properties and load shown in that figure, the first natural frequency and the fourth node static deflection are $f_{1}=\omega_{1} / 2 \pi=24.283 \mathrm{~Hz}$ and $u_{4}=12.213 \mathrm{~mm}$. The first mode is also computed, and the modal node deflections are $\phi_{9,1}=0.30271, \phi_{15,1}=0.80156$, $\phi_{21,1}=1.000, \phi_{27,1}=0.68719$, where NASTRAN DOF numbering is used. Two types of problems are considered: correlation with respect to measured values of $f_{1}^{\prime}$ and $u_{4}^{\prime}$ and correlation with respect to $f_{i}^{\prime}$ and the corresponding mode, where incomplete modal data are available. In all beam applications, 8 modes are extracted, $n_{r}=8 ; 10$ correlation variables are used, the moment of inertia and cross section area of each element; the accuracy used in RESTRUCT in satisfying static constraints and diagonal dynamic constraints-Eqs. (36) and (33)-is $\epsilon_{D}=10^{-6}$; and the accuracy used in satisfying admissibility (orthogonality) constraints-Eqs. (34) and (35)-is $\epsilon_{A}=10^{-3}$. 

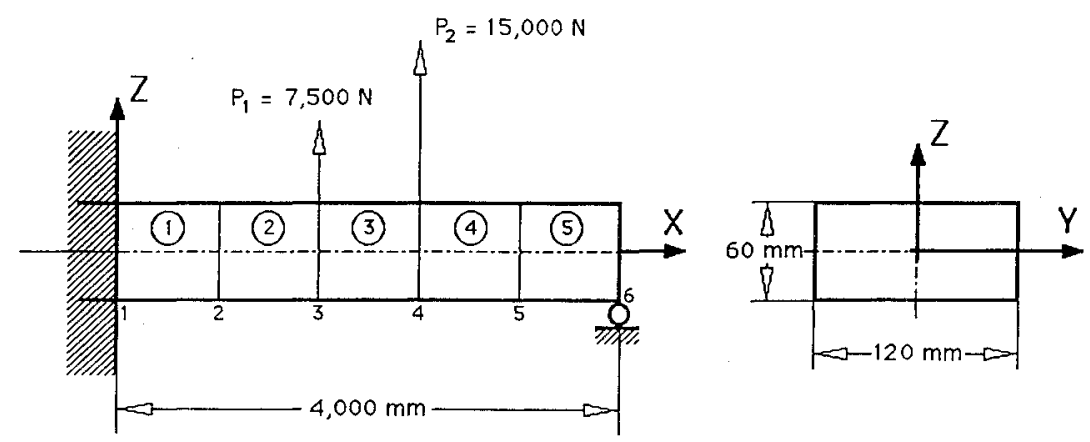

$$
\text { Properties : } \begin{aligned}
E & =2.07 \cdot 10^{5} \mathrm{MPa} \\
e & =7.833 \cdot 10^{-9} \mathrm{NSec}^{2} / \mathrm{mm}^{4} \\
I_{y} & =2.160 \cdot 10^{6} \mathrm{~mm}^{4} \\
I_{z} & =8.640 \cdot 10^{6} \mathrm{~mm}^{4} \\
v & =0.3
\end{aligned}
$$

\begin{tabular}{|c|c|c|c|c|c|c|c|c|c|c|}
\hline \multirow[b]{3}{*}{ Case no. } & \multicolumn{2}{|c|}{$\begin{array}{c}\text { Correlation } \\
\text { measures }\end{array}$} & \multicolumn{4}{|c|}{ Six admissibility constraints } & \multicolumn{4}{|c|}{ Twelve admissibility constraints } \\
\hline & \multirow[b]{2}{*}{$r_{\omega}^{2}$} & \multirow[b]{2}{*}{$r_{u}$} & \multicolumn{2}{|c|}{ Reanalysis } & \multicolumn{2}{|c|}{ Error, $\% 0^{a}$} & \multicolumn{2}{|c|}{ Reanalysis } & \multicolumn{2}{|c|}{ Error, $\%$} \\
\hline & & & $r_{\omega} 2$ & $r_{u}$ & $r_{\omega} 2$ & $r_{u}$ & $r_{\omega^{2}}$ & $r_{u}$ & $r_{\omega}{ }^{2}$ & $r_{u}$ \\
\hline 1 & 1.4595 & 0.6852 & 1.4583 & 0.6884 & 0.08 & 0.47 & 1.4594 & 0.6881 & -0.01 & 0.42 \\
\hline 2 & 2.0000 & 0.5000 & 1.9937 & 0.5034 & 0.32 & 0.68 & 2.0000 & 0.5021 & 0.0 & 0.42 \\
\hline 3 & 1.4595 & 1.4595 & 1.4496 & 1.4742 & -0.68 & 1.01 & 1.4594 & 1.4649 & -0.01 & 0.37 \\
\hline 4 & 2.0000 & 2.0000 & 1.9633 & 2.0423 & 1.84 & 2.12 & 2.0000 & 2.0075 & 0.0 & 0.38 \\
\hline 5 & 0.6851 & 1.4595 & 0.6847 & 1.4658 & -0.04 & 0.43 & 0.6851 & 1.4649 & 0.0 & 0.37 \\
\hline 6 & 0.5000 & 2.0000 & 0.4989 & 2.0112 & -0.22 & 0.56 & 0.5000 & 2.0073 & 0.0 & 0.37 \\
\hline 7 & 0.7071 & 0.7071 & 0.7036 & 0.7138 & -0.49 & 0.95 & 0.7071 & 0.7108 & 0.0 & 0.52 \\
\hline 8 & 0.5000 & 0.5000 & 0.4908 & 0.5108 & -1.84 & 2.16 & 0.5000 & 0.5026 & 0.0 & 0.52 \\
\hline
\end{tabular}

Response : $f_{1}=\frac{\omega_{1}}{2 \pi}=24.283 \mathrm{~Hz}$

$$
u_{4}=12.213 \mathrm{~mm}
$$

Fig. 4 Clamped-hinged beam model: five elements, nine DOFs.

Table 1 Correlation of beam model in bending: frequency and static deflection measures

Initial model predictions:

Number of extracted modes:

$f_{1}=24.2832 \mathrm{~Hz}, u_{4}=12.2126 \mathrm{~mm}$

Number of correlation variables: $p=10$

Correlation measure ratios: $\quad r_{\omega 2}=\omega^{\prime 2} / \omega_{1}^{2}, r_{u}=u_{4}^{\prime} / u_{4}$

Total CPU time, $t: \quad S_{\alpha}=6, t \cong 18,700 \mathrm{~ms}$

$S_{a}=12, t \cong 20,600 \mathrm{~ms}$

aValues predicted by RESTRUCT were equal to measured values.

\section{Correlation Measures: $f_{1}^{\prime}$ and $u_{4}^{\prime}$}

Sixteen applications were run and results are summarized in Table 1 . Specifically, eight cases were run with the admissibility domain defined in the correction phase by 6 or 12 constraints. Those eight cases correspond to all combinations of 46 or $100 \%$, increase or decrease in $\lambda_{1}=\omega_{1}^{2}$ or $u_{4}$. We can draw the following conclusions from Table 1 . When six admissibility constraints are used, $S_{a}=6$, the total number of constraints in the correction phase is $S=S_{a}+2=8$, which is less than the number of correlation variables, $p=10$. Thus, the problem is solved by optimization. When $S_{a}=12, S=S_{a}+$ $2=14>p=10$, and the problem is solved by the generalized inverse algorithm. In all 16 applications, the relative error is small. It appears that the results of any overdetermined case are better than the corresponding underdetermined case. This occurs for two reasons. First, six admissibility constraints are not enough to define properly the admissible domain. Second, 12 admissibility constraints define well the admissible domain, and within the selected computational acuracy all $S=14$ constraints are satisfied. In fact, when $S_{a}=8, S=S_{a}+2=$ $10=p$, and the results are as accurate as in the overdetermined case.

\section{Conflicting Requirements}

Increasing $f_{1}$ or decreasing $u_{4}$ induces increased structural stiffness. Opposite action decreased structural stiffness. In applications $1,2,5$, and 6 , both frequency and static deflection correlation measures have compatible effects on IM. In applications $3,4,7$, and 8 , correlation measures have conflicting effects. By comparing cases 1 and 3,2 and 4, 5 and 7, and 6 and 8 , we see that compatible requirements are easier to satisfy.

Further, as expected, it is easier to satisfy changes in modal dynamic and static correlation measures of $46 \%$ rather than $100 \%$. For small-scale structures, like the beam problem considered in this section, satisfaction of changes by a factor of 4 is possible with high accuracy. Such applications, however, are of no interest in model correlation. In general, it is more difficult to achieve as high accuracy in larger scale problems, like the offshore tower considered in Secs. IV.B, V, and VI.

\section{Cognate Space}

The beam problem considered here is studied only in bending in the $(x, z)$ plane. Thus, all extracted modes are cognate. This is verified numerically by the magnitude of the admixture coefficients. All $c_{i j}$ are much greater than $10^{-6}$, which is the cut-off point in RESTRUCT for eliminating modes that have a minor effect in Eq. (26). In the correction phase solution, all Lagrange multipliers are nonzero, which indicates that the entire cognate admissible space is active. This was anticipated since all modes are in the same plane. 
Table 4 Correlation of offshore tower model: bending frequency and static deflection measures

\begin{tabular}{|c|c|c|c|c|c|c|c|c|c|c|c|}
\hline \multirow[b]{2}{*}{ Case no. } & \multicolumn{2}{|c|}{$\begin{array}{c}\text { Correlation } \\
\text { measures }\end{array}$} & \multirow[b]{2}{*}{$n_{r}$} & \multirow[b]{2}{*}{$S_{a}$} & \multicolumn{2}{|c|}{ Prediction } & \multicolumn{2}{|c|}{ Reanalysis } & \multicolumn{2}{|c|}{ Error, $\%_{0}{ }^{a}$} & \multirow[b]{2}{*}{$\mathrm{CPU}, \mathrm{ms}$} \\
\hline & $r_{\omega}^{2}$ & $r_{u}$ & & & $r_{\omega_{L}^{2}}$ & $r_{u}$ & $r_{\omega_{1}^{2}}^{2}$ & $r_{u}$ & $r_{\omega_{1}^{2}}^{2}$ & $r_{u}$ & \\
\hline 1 & \multirow[t]{2}{*}{1.5006} & \multirow[t]{2}{*}{0.647} & 13 & 8 & \multirow[t]{2}{*}{1.5006} & 0.648 & 1.4616 & 0.6610 & 2.60 & -2.06 & 440,340 \\
\hline 2 & & & 16 & 12 & & 0.648 & 1.4689 & 0.6582 & 2.11 & -1.57 & 660,516 \\
\hline 3 & \multirow[t]{4}{*}{1.9668} & \multirow[t]{4}{*}{0.484} & 5 & 0 & \multirow{4}{*}{1.9668} & 0.4868 & 1.7730 & 0.5268 & 9.85 & -8.22 & -- \\
\hline 4 & & & 10 & 4 & & 0.4867 & 1.7994 & 0.5190 & 8.51 & -6.64 & $\ldots$ \\
\hline 5 & & & 13 & 8 & & 0.4867 & 1.8358 & 0.5113 & 6.66 & -5.05 & 733,749 \\
\hline 6 & & & 16 & 12 & & 0.4867 & 1.8648 & 0.5046 & 5.19 & -3.68 & 976,567 \\
\hline 7 & \multirow[t]{2}{*}{1.5006} & \multirow{2}{*}{1.5007} & 13 & 8 & \multirow{2}{*}{1.5006} & 1.5059 & 1.4759 & 1.5190 & 1.65 & -1.20 & 445,723 \\
\hline 8 & & & 16 & 12 & & 1.5059 & 1.4649 & 1.5285 & 2.38 & -1.50 & 672,591 \\
\hline 9 & \multirow[t]{4}{*}{1.9668} & \multirow[t]{4}{*}{1.9672} & 5 & 0 & \multirow[t]{4}{*}{1.9668} & 1.9756 & 1.7128 & 2.2594 & 14.94 & -14.36 & - \\
\hline 10 & & & 10 & 4 & & 1.9758 & 1.8714 & 2.0681 & 4.85 & -4.67 & -— \\
\hline 11 & & & 13 & 8 & & 1.9759 & 1.8767 & 2.0335 & 4.58 & -2.92 & 733,850 \\
\hline 12 & & & 16 & 12 & & 1.9759 & 1.8525 & 2.0581 & 5.81 & -4.16 & 979,056 \\
\hline 13 & \multirow[t]{2}{*}{0.6473} & \multirow[t]{2}{*}{0.647} & 13 & 8 & \multirow[t]{2}{*}{0.6473} & \multirow[t]{2}{*}{0.6482} & 0.6372 & 0.6655 & 1.56 & -2.67 & 453,090 \\
\hline 14 & & & 16 & 12 & & & 0.6276 & 0.6769 & 3.04 & -4.43 & 582,174 \\
\hline 15 & \multirow[t]{2}{*}{0.4843} & \multirow[t]{2}{*}{0.484} & 13 & 8 & \multirow[t]{2}{*}{0.4843} & \multirow[t]{2}{*}{0.4868} & 0.4625 & 0.513 & -4.05 & 5.99 & 733,849 \\
\hline 16 & & & 16 & 12 & & & 0.4771 & 0.5358 & -7.68 & 10.70 & 969,570 \\
\hline \multirow{3}{*}{\multicolumn{4}{|c|}{$\begin{array}{l}\text { Initial model predictions: } \\
\text { Number of correlation variables: } \\
\text { Correlation measure ratios: }\end{array}$}} & \multirow{3}{*}{\multicolumn{5}{|c|}{$\begin{array}{l}f_{1}=0.7472 \mathrm{~Hz}, u_{11}=0.0578 \mathrm{~m} \\
p=10 \\
r_{\omega_{1}^{2}}=\omega_{1}^{\prime 2} / \omega_{1}^{2}, r_{u}=u_{11}^{\prime} / u_{11}\end{array}$}} & & & \\
\hline & & & & & & & & & & & \\
\hline & & & & & & & & & & & \\
\hline
\end{tabular}

${ }^{a}(a-b) / a ; a$ is computed by RESTRUCT, $b$ is computed by UM, using MSC/NASTRAN.

Table 5 Correlation of offshore tower model: torsion frequency measure

\begin{tabular}{|c|c|c|c|c|c|c|c|}
\hline \multirow[b]{2}{*}{ Case no. } & \multirow{2}{*}{$\frac{\begin{array}{c}\text { Correlation } \\
\text { measures }\end{array}}{r_{\omega}^{2}}$} & \multirow[b]{2}{*}{$n_{r}$} & \multirow[b]{2}{*}{$S_{a}$} & \multirow[b]{2}{*}{ Predicted } & \multirow{2}{*}{$\frac{\text { Reanalysis }}{r_{\omega^{2}}}$} & \multirow{2}{*}{$\frac{\text { Error, } 00^{\mathrm{a}}}{r_{\omega^{2}}}$} & \multirow[b]{2}{*}{$\mathrm{CPU}, \mathrm{ms}$} \\
\hline & & & & & & & \\
\hline 1 & 1.3064 & 19 & 4 & 1.3064 & 1.2952 & 0.86 & 177,589 \\
\hline 2 & 1.5964 & 19 & 4 & 1.5964 & 1.5564 & 2.51 & 306,703 \\
\hline 3 & 1.9508 & 19 & 4 & 1.9508 & 1.8562 & 4.85 & 439,472 \\
\hline \multicolumn{3}{|c|}{$\begin{array}{l}\text { Initial model prediction: } \\
\text { Number of correlation variables: } \\
\text { Correlation measure ration: }\end{array}$} & \multicolumn{2}{|c|}{$\begin{array}{l}f_{3}=0.852 \mathrm{~Hz} \\
p=12 \\
r_{\omega^{2}}=\omega_{3}^{\prime 2} / \omega_{3}^{2}\end{array}$} & & & \\
\hline
\end{tabular}

${ }^{a}(a-b) / a ; a$ is computed by RESTRUCT, $b$ is computed by UM, using MSC/NASTRAN.

Table 6 Correlation of offshore tower model: bending and torsion frequency measured

\begin{tabular}{|c|c|c|c|c|c|c|c|c|c|c|c|}
\hline \multirow[b]{2}{*}{ Case no. } & \multicolumn{2}{|c|}{$\begin{array}{c}\text { Correlation } \\
\text { measures }\end{array}$} & \multirow[b]{2}{*}{$n_{r}$} & \multirow[b]{2}{*}{$S_{a}$} & \multicolumn{2}{|c|}{ Prediction } & \multicolumn{2}{|c|}{ Reanalysis } & \multicolumn{2}{|c|}{ Error, $\% 0^{a}$} & \multirow[b]{2}{*}{$\mathrm{CPU}, \mathrm{ms}$} \\
\hline & $r_{\omega_{1}^{2}}^{2}$ & $r_{\omega_{3}^{2}}^{2}$ & & & $r_{\omega_{1}^{2}}^{2}$ & $r_{\omega \frac{2}{3}}$ & $r_{\omega_{1}^{2}}$ & $r_{\omega}{ }_{3}^{2}$ & $r_{\omega_{1}^{2}}^{2}$ & $r_{\omega_{3}^{2}}$ & \\
\hline 1 & 1.45345 & 1.4830 & 19 & 8 & 1.4534 & 1.4830 & 1.4244 & 1.4560 & 2.00 & 1.82 & 934,210 \\
\hline 2 & 1.8649 & 1.9286 & 16 & 12 & 1.8635 & 1.9279 & 1.7736 & 1.8305 & 4.82 & 5.05 & $1,122,017$ \\
\hline 3 & 1.8649 & 1.9286 & 19 & 8 & 1.8635 & 1.9279 & 1.7685 & 1.8385 & 5.10 & 4.64 & $1,556,700$ \\
\hline \multicolumn{4}{|c|}{$\begin{array}{l}\text { Initial model predictions: } \\
\text { Number of correlation variables: } \\
\text { Correlation measure ratios: }\end{array}$} & \multicolumn{7}{|c|}{ 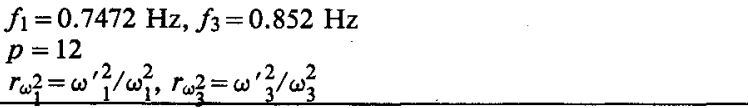 } & \\
\hline
\end{tabular}

a $(a-b) / a ; a$ is computed by RESTRUCT, $b$ is computed by UM, using MSC/NASTRAN.

indeed this is the case, and all $c_{i j}$ relating mode 1 to modes not belonging to $J_{C}$ are less than $10^{-6}$. Solution of the optimization problem in the correction phase shows that only Lagrange multipliers associated with bending modes in the $(x, y)$ plane are nonzero. That is, the active admissible cognate subspace consists of orthogonality conditions $(1,8),(1,12),(1,15)$, with respect to stiffness and mass matrices.

\section{Correlation in Torsion}

The offshore tower model described in Sec. IV.B is used in this application. There is only one measured quantity, $\omega_{3}^{\prime}$. Thus, only one correlation measure is used, $r_{\omega_{3}^{2}}=\lambda_{3}^{\prime} / \lambda_{3}=\omega_{3}^{\prime 2} /$ $\omega_{3}^{2}$. Three cases are run for changes by factors of 1.3, 1.6, 1.95; the results are summarized in Table 5. To analyze the results, the following information is needed. As mentioned in Sec. IV.B, $J_{T}=\{3,18,19\}$. Further, $J_{M}=\{3\}, J_{C}=J_{T}, J_{C_{3}}=$ $J_{C_{18}}=J_{C_{19}}=J_{T}$. Now, we can draw the following conclusions.
1) The admissible cognate space includes only four orthogonality conditions, that is, $(3,18),(3,19)$, with respect to stiffness and mass matrices.

2) The set of available measurements is inadequate for accurate model correlation, because it provides only five constraints in each incremental correction phase. Specifically, there are four orthogonality and one frequency constraints. Nonetheless, correlation is very satisfactory for a $30 \%$ change in $\lambda_{3}=\omega_{3}^{2}$, acceptable for a $60 \%$ change, and approximate for a $90 \%$ change.

5) At least 19 modes must be extracted to have at least three cognate modes in $J_{C}$.

In the active admissible cognate subspace, the cognate space is composed only of three torsional modes, 3, 18, 19. Numerical computations show that indeed all $C_{i j}$ relating mode 3 to a mode not belonging to $J_{C}$ are less than $10^{-6}$. Solution of the optimization problem in correction phase shows that all La- 
grange multipliers of admissibility conditions are nonzero. This implies that the entire admissible cognate space is active.

\section{Correlation in Bending and Torsion}

The offshore tower model described in Sec. IV.B is used in this application as well. Two quantities are measured in this correlation problem, $\omega_{1}^{\prime}$ and $\omega_{3}^{\prime}$. Thus, two correlation measures are used:

$$
r_{\omega \mathrm{f}}=\lambda_{1}^{\prime} / \lambda_{1}=\omega_{1}^{2} / \omega_{1}^{2}, \quad r_{\omega_{3}^{2}}=\lambda_{3}^{\prime} / \lambda_{3}=\omega_{3}^{\prime 2} / \omega_{3}^{2}
$$

Three cases are run for changes by factors of about 1.5 and 1.9 , and results are summarized in Table 6 . In this problem, since data on one bending and one torsional modes are available, we can define the following sets:

$$
\begin{gathered}
J_{M}=\{1,3\}, J_{C}=J_{B}+J_{T} \\
J_{C_{1}}=J_{C_{2}}=J_{C_{8}}=J_{C_{9}}=J_{C_{12}}=J_{C_{13}}=J_{C_{15}}=J_{C_{16}}=J_{B} \\
J_{C_{3}}=J_{C_{18}}=J_{C_{19}}=J_{T}
\end{gathered}
$$

1) The admissible cognate space includes more orthogonality conditions than $p$, the number of correlation variables. To make the problem in the correction phase underdetermined, either more correlation variables should be introduced or fewer orthogonality conditions used.

2) Changes by a factor of about 1.5 are treated adequately by RESTRUCT. Correlation with respect to changes by a factor of 2 are only approximate. Obviously, the set of measured data is not adequate.

3) Comparing cases 2 and 3, we see that, even though fewer constrains are used in case 3 , results in $\omega_{3}^{\prime}$ are better because the proper set of admissibility constraints was selected. Specifically, in case 2 , only bending orthogonality constraints are used because 16 modes were extracted, thus excluding torsional modes 18 and 19. In case 3, four bending and four torsional orthogonality constraints were used.

In the active admissible cognate subspace, the cognate space is composed of eight bending and three torsional modes, that is $J_{C}=\{1,2,8,9,12,13,15,16 ; 3,18,19\}$. Numerical computations verify that all $C_{i j}$ relating mode 1 to any mode not belonging to $J_{B}$ are $<10^{-6}$. Similarly, all $C_{i j}$ relating mode 3 to any mode not belonging to $J_{T}$ are $<10^{-6}$. Solution of the optimization problem in correction phase shows that Lagrange multipliers associated with orthogonality conditions between mode 1 and any bending mode in the $(y, z)$ plane with respect to either $[k]$ or $[m]$ are zero. That is, the active admissible cognate subspace includes only orthogonality conditions between torsional modes and between bending modes in the $(x, y)$ plane.

\section{Conclusions}

The problem of correlating an FEM to a real structure for which modal vibration and static deflection measurements are available was studied. The set of modal measurements may be incomplete and only parts of specific modes may have been measured. Measurements are assumed to be exact. In the problem formulation, it was assumed that large differences may exist between measured static and/or dynamic response and FEA predictions by the initial model (IM). Differences as large as 100-300\% were used in the numerical applications to account for structures built to large manufacturing tolerances, like marine structures. A perturbation based methodology was developed to solve the problem without trial and error and without additional FEAs. Both mass and stiffness matrices were updated to produce an updated (correlated) model (UM). Correlation variables were stiffness, mass, or geometric properties of structural elements or groups of elements, and not individual terms in the stiffness and mass matrices. Thus, the updated model mass and stiffness matrix distribution corre- sponded to a real structure. The resulting static and dynamic general perturbation equations were implicit nonlinear expressions of the correlation variables. The developed solution algorithm was incremental and involved three phases. In the incremental prediction phase, linear inadmissible predictions were made based on an incomplete set of modal measurements. In the intermediate phase-used only in the first increment-the space cognate to the measured modes was identified so that modal data which were not measured could be computed at reduced cost in the space of cognate rather than extracted modes. In the third incremental phase, admissible corrections were made to ensure that the produced UM of a real structure has mass and stiffness matrices orthogonal to the modal set, which was partially measured and partially calculated. The Lagrange multipliers indicated that some of the orthogonality constraints in the admissible cognate subspaces may be inactive. The resulting problems in both prediction and correction phases in each increment may be underdetermined or overdetermined. In the former case, results were more accurate and were produced by a quadratic programming algorithm. Overdetermined problems were solved by a minimum error algorithm in satisfaction of measured data and admissibility constraints. The user of code RESTRUCT is informed accordingly, since an overdetermined problem may indicate incorrect measurements or conflicting correlation requirements. The methodology was initially developed for redesign problems, and was applied to structures as large as 1254 DOFs. ${ }^{16} \mathrm{~A}$ new algorithm was developed to solve the model correlation problem, and RESTRUCT was modified to implement it. A 5-element 9-DOF beam model, and a 104-element 192-DOF offshore tower model were used to test the correlation algorithm in bending, torsion, and bending and torsion. Conflicting requirements, admissibility cognate space identification, and activity of constraints in cognate subspaces were investigated numerically.

During the review process of this paper, two important issues in structural modeling and design were raised by the reviewers. Those issues are presented below for the benefit of the readers, completeness of this paper, and for suggesting new research avenues.

1) In the algorithm developed in this paper, the designer specifies the properties of elements or groups of elements that are allowed to change (correlation variables) and the computer returns values of those properties. Those values are optimal, according to some selected criterion. The issue here is, which is the most appropriate set of correlation variables to produce a physically correct model. (The authors would like to add that the outcome of the correlation process also depends on the optimality criterion.) This issue reveals at the same time a strength and a limitation of the developed methodology. It has raised the design modeling problem by one level. Specifically, the problem now is how to identify structural properties (geometry, stiffness, mass) whose values are uncertain and mostly affect measured data, rather than computing their values; the latter is treated in this paper. One way to address this new design question is by finding numerically which structural elements contribute mostly to the modal energy in the subspace cognate to measured data. This research topic is currently pursued by the authors.

At this point, it is important to bring up the following issue, which places into perspective model correlation. Almost no mathematical model in mechanics is capable of modeling the corresponding system correctly over a broad range of parameters. Models are made to reproduce only a specific response. For instance, a simple mass-spring-dashpot system is modeled properly by $m \ddot{x}+c \dot{x}+k x=f(t)$ only near resonance. Away from resonance, effects like nonlinear damping, mass of spring, etc., render the above model inaccurate. In this simple example, the designer may select as correlation variables $m, c$, $k$, or any combination of those in order to make the model predict measured data. Then, he may expect his model to be satisfactory in the vicinity of the phenomenon it describes. He 
should also expect poor prediction away from that phenomenon, not because the correlation process is inadequate, but because the model is not applicable. At the present state of the art, the correlation process can compute values for the selected correlation variables. The designer's new task becomes the selection of the correlation variables among $m, c$, and $k$. In the case of the offshore tower used in numerical applications in this paper, if the FEM is correlated only with respect to first mode data (frequency, mode shape), it will predict satisfactorily the tower's dynamic response only if the response spectrum is a narrow band around the first frequency. If that is not expected to be the case, and there is significant energy in other modes, pertinent measurements should be introduced in the correlation process.

2) A strong point of the correlation methodology developed in this paper is that the correlation variables represent properties of structural elements, or groups of elements, and not simply terms in $[k]$ and $[m]$. The issue raised by a reviewer is, "Once a model is reduced from the finite element analysis size of tens of thousands of DOFs to the size required for dynamic analyses (500-1000 DOF) individual element stiffness matrices are nearly impossible to identify." The approach that we follow for redesign and model correlation for large-scale structure is based on substructuring. Individual element stiffness matrices can still be identified. This method is pursued for redesign of submarine hulls for the Office of Naval Research.

\section{Acknowledgments}

This publication has been partly sponsored by The University of Michigan/Sea Grant/Industry Constortium in Offshore Engineering under Michigan Sea Grant College Program, projects E/GLE-14 and R/T-23, under Grant NA85AA-D-SG045C from the Office of Sea Grant, National Oceanic and Atmospheric Administration (NOAA), U.S. Department of Commerce, and funds from the State of Michigan. Industry participants include the American Bureau of Shipping; ARCO Gas and Oil Company (1988-); Conoco, Inc.; Exxon Production Research; Friede and Goldman, Ltd.; Noble, Denton \& Associates, Inc. (1985-1986); Shell Companies Foundation (1985-1986); and the U.S. Coast Guard (1986-). The U.S. Government is authorized to produce and distribute reprints for governmental purpose notwithstanding any copyright notation appearing hereon.

\section{References}

${ }^{1}$ Berman, A., and Nagy, E. J., "Improvement of a Large Analytical Model Using Test Data," AIAA Journal, Vol. 21, No. 8, 1983, pp. 1168-1173.

${ }^{2}$ Kabe, A. M., "Stiffness Matrix Adjustment Using Mode Data," AIAA Journal, Vol. 23, No. 9, 1985, pp. 1431-1436.

${ }^{3}$ Fuh, J. S., and Berman, A.," "Comment on "Stiffness Matrix Adjustment Using Mode Data,' " AIAA Journal, Vol. 16, No. 11, 1978, pp. 1208-1210.

${ }^{4}$ Baruch, M., "Optimization Procedure to Correct Stiffness and Flexibility Matrices Using Vibration Tests," AIAA Journal, Vol. 16, No. 11, 1978, pp. 1208-1210.

${ }^{5}$ Wei, F.-S., and Zhang, D.-W., "Mass Matrix Modification Using Element Correction Method," AIAA Journal, Vol. 27, No. 1, 1989, pp. 119-121.

${ }^{6}$ Berman, A., "Mass Matrix Correction Using an Incomplete Set of Measured Modes," AIAA Journal, Vol. 17, No. 10, 1979, pp. 1147-1148.

${ }^{7}$ Taylor, J. E., "Scaling a Discrete Structural Model to Match Measured Modal Frequencies," AIAA Journal, Vol. 15, No. 11, 1977, pp. 1647-1649.

${ }^{8}$ Caesar, B., and Peter, J., "Direct Update of Dynamic Mathematical Models from Modal Test Data," AIAA Journal, Vol. 25, No. 11, 1987, pp. 1494-1499.
${ }^{9}$ Chen, J. C., and Garba, J. A., "Analytical Model Improvement Using Modal Test Results," AIAA Journal, Vol. 18, No. 6, 1980, pp. 684-690.

${ }^{10}$ Chen, S. Y., and Fuh, J. S., " Application of Generalized Inverse in Structural System Identification," AIAA Journal, Vol. 22, No. 12, 1984 , pp. $1827-1828$.

${ }^{11}$ Targoff, W. P., "Orthogonality Check and Correction of Measured Modes," AIAA Journal, Vol. 14, No. 2, 1976, pp. 164-167.

${ }^{12}$ Baruch, M., and Bar Itzhack, I. Y., "Optimal Weighted Orthogonalization of Measured Modes," AIAA Journal, Vol. 16, No. 4, 1978, pp. 346-351.

${ }^{13}$ Collins, J. D., Hart, G. C., Hasselman, T. K., and Kennedy, B., "Statistical Identification of Structures," AIAA Journal, Vol. 12, No. 2, 1974, pp. 185-190.

${ }^{14}$ Stetson, K. A., "Perturbation Method of Structural Design Relevant to Holographic Vibration Analysis," AIAA Journal, Vol. 13, No. 4, 1975, pp. 457-459.

${ }^{15}$ Stetson, K. A., and Harrison, I. R., "Redesign of Structural Vibration Modes by Finite-Element Inverse Perturbation," ASME Transactions, Journal of Engineering for Power, Vol. 103, No. 2, 1981, pp. 319-325.

${ }^{16}$ Sandström, R. E., and Anderson, W. J., "Modal Perturbation Methods for Marine Structures," Transactions of the Society of Naval Architects and Marine Engineers, Vol. 90, 1982, pp. 41-54.

${ }^{17}$ Hoff, C. J., Bernitsas, M. M., Sandström, R. E., and Anderson, W. J., "Nonlinear Incremental Inverse Perturbation Method for Structural Redesign of Risers," Proceedings of AIAA/ASME/ ASCE/AHS 24th Structures, Structural Dynamics and Materials Conference, AIAA, New York, May 1983; also AIAA Journal, Vol. 22, No. 9, 1984, pp. 78-85.

${ }^{18}$ Hoff, C. J., and Bernitsas, M. M., "Dynamic Redesign of Marine Structures," Journal of Ship Research, Vol. 29, No. 4, 1985, pp. 285-295.

${ }^{19}$ Hoff, C. J., and Bernitsas, M. M., "Static Redesign of Offshore Structures," Proceedings of 5th International Offshore Mechanics and Arctic Engineering Symposium, Vol. III, Tokyo, Japan, American Society of Mechanical Engineers, New York, 1986, pp. 78-85.

${ }^{20}$ Bernitsas, M. M., Hoff, C. J., and Kokarakis, J. E., "Nonlinear Inverse Perturbation in Structural Redesign of Risers," Proceedings of the 3rd Offshore Mechanics and Arctic Engineering Symposium, New Orleans, LA, Feb. 12-14, 1984; also, ASME Transactions, Journal of Energy Resources Technology, Vol. 107, No. 2, 1985, pp. 256-263.

${ }^{21} \mathrm{Kim}, \mathrm{K}$. O., and Anderson W. J., "Dynamic Condensation in Structural Dynamics Redesign," Proceedings of 7 th International Symposium on the Computational Aspects of the Finite Element Method, Chicago, IL, Aug. 1983; AIAA Journal, Vol. 22, No. 11, 1984, pp. 1616-1617.

${ }^{22}$ Gans, H. D., and Anderson, W. J., "Structural Optimization Incorporating Centrifugal and Coriolis Effects," Proceedings of the 30th AIAA/ASME/ASCE/AHS/ASC Structures, Structural Dynamics and Materials Conference, AIAA, Washington, DC, April 1989, pp. 1311-1320.

${ }^{23} \mathrm{Kim}$, J. H., and Bernitsas, M. M., "Redesign of Marine Structures," Journal of Marine Structures, Vol. 1, No. 2, 1988, pp. 139-183.

${ }^{24}$ Haftka, R. T., and Prasad, B., "Programs for Analysis and Resizing of Complex Structures," Computers and Structures, Vol. 10, No. 2, 1979, pp. 323-330.

${ }^{25}$ Haug, E. J., Choi, K. K., and Komkov, V., Design Sensitivity Analysis of Structural Systems, Academic, New York, 1986.

${ }^{26}$ Bernitsas, M. M., and Kim, J., "RESTRUCT: A Program for Redesign of Structures," Rept. to Univ. of Michigan/Sea Grant/Industry Consortium in Offshore Engineering and Publication No. 303, Dept. of Naval Architecture and Marine Engineering, Univ. of Michigan, Ann Arbor, MI, Sept. 1987.

${ }^{27}$ Pickett, R. M., Jr., Rubinstein, M. F., and Nelson, R. B., "Automated Structural Synthesis Using a Reduced Number of Design Coordinates," AIAA Journal, Vol. 11, No. 4, 1973, pp. 489-494.

${ }^{28}$ Luenberger, D. G., Introduction to Linear and Nonlinear Programming, Addison-Wesley, Reading, MA, 1973.

${ }^{29}$ Gill, P. E., Murray, W., Saunders, M. A., and Wright, M. H., "User's Guide for SOL/QPSOL: A Fortran Package for Quadratic Programming," Dept. of Operations Research, Stanford Univ., Stanford, CA, 1983. 